\title{
Segurança do Paciente na Administração de Quimioterapia Antineoplásica: uma Revisão Integrativa
}

Patient Safety in Administering Antineoplastic Chemoteraphy: an Integrative Review

\author{
Seguridad de los Pacientes en la Administración de la Quimioterapia Antineoplásica: \\ una Revisión Integrativa
}

\author{
Talita dos Santos Ribeiro ${ }^{1}$; Valdete Oliveira Santos ${ }^{2}$
}

\section{Resumo}

Introduçáo: A segurança do paciente pode ser definida como a redução a um mínimo aceitável do risco de um dano desnecessário ocorrer associado aos cuidados em saúde. Os erros em uma ou mais etapas no processo de quimioterápicos podem ter consequências graves para os pacientes, desde toxicidades inesperadas até o óbito. Objetivo: Identificar as estratégias recomendadas na literatura que visam à segurança do paciente na administração de quimioterapia antineoplásica. Método: Trata-se de uma revisão integrativa da literatura que utilizou como critérios de inclusão: publicaçôes em português, inglês e espanhol, disponíveis gratuitamente nas bases de dados CINAHL e PUBMED em textos completos no período de 2000 a 2013. Resultados: A busca resultou em 1.385 publicaçóes, sendo selecionadas 13 publicaçôes científicas internacionais que atendiam ao objetivo do estudo. Discussão: Nos artigos analisados, os autores enfatizam os protocolos, procedimentos e o processo de administração de quimioterapia, estabelecendo condutas únicas, de forma clara, para a manutenção da segurança em todas as etapas. Conclusão: A utilização de boas práticas não é suficiente para evitar que falhas ou erros na administração de quimioterápicos ocorram. Torna-se necessária a criação de uma cultura de segurança voltada para o compartilhamento da responsabilidade e a implementaçáo de políticas e normas institucionais, a fim de melhorar a segurança, ambas elaboradas a partir de uma equipe multiprofissional com características interdisciplinares.

Palavras-chave: Antineoplásicos; Erros de Medicação; Enfermagem Oncológica; Segurança do Paciente; Revisão

\footnotetext{
${ }^{1}$ Enfermeira. Pós-graduada pelo Programa de Residência Multiprofissional em Oncologia do Instituto Nacional de Câncer José Alencar Gomes da Silva (INCA). Rio de Janeiro (RJ), Brasil.E-mail: talita.unirio@gmail.com.

${ }^{2}$ Enfermeira. Doutoranda pela Universidade Federal do Estado do Rio de Janeiro (Unirio). Programa de Pós-Graduação em Enfermagem e Biociências (PPGENFBIO). Mestre em Enfermagem pela Escola de Enfermagem Anna Nery da Universidade Federal do Rio de Janeiro (EEAN/UFRJ). Chefe de Serviço em Procedimentos Externos do Hospital do Câncer I (HCI)/INCA. Integrante do Grupo de Orientadores do Curso de Residência Multiprofissional em Oncologia e Docente da Residência Multiprofissional em Oncologia. Rio de Janeiro (RJ), Brasil. E-mail: vsantos@inca.gov.br.

Endereço para correspondência: Talita dos Santos Ribeiro. Rua Herman Lima, s/n, quadra C, lote 03 - Senador Augusto Vasconcelos. Campo Grande (RJ), Brasil. CEP: 23.013-345. E-mail: talita.unirio@gmail.com.
} 


\section{INTRODUÇÃO}

Os estudos baseados na segurança do paciente ainda são recentes, tanto em âmbito nacional como internacional. Passaram a apresentar evidência no cenário internacional após a publicação pelo Instituto de Medicina dos Estados Unidos, no ano de 1999, do relatório intitulado To Err is Human: Building a Safer Health Care System, apresentando à sociedade e à comunidade científica estudos epidemiológicos nos quais estimaram que anualmente entre 44.000 a 98.000 norte-americanos morriam em decorrência de erros que acontecem no sistema de saúde ${ }^{1}$.

A variedade de conceitos relacionados à segurança do paciente fez com que a Organização Mundial da Saúde (OMS) instituísse uma comissáo composta por especialistas que buscassem um consenso, criando uma Classificação Internacional para Segurança do Paciente (International Classification for Patient for Patient Safety - ICPS) com a proposta de melhorar o conhecimento e a transferência de informaçôes. De acordo com a ICPS, "segurança do paciente é reduzir a um mínimo aceitável, o risco de dano desnecessário associado ao cuidado de saúde"2.

Estima-se que uma em cada dez admissóes hospitalares resultarão em incidentes que poderiam ser evitados, sendo que um a cada três incidentes acarretam comprometimento real ao paciente. Segundo a ICPS, incidente é o evento ou circunstância que poderia ter resultado em dano desnecessário ao paciente, enquanto o near miss é definido como o incidente sem lesão que atingiu o paciente, e evento adverso como o incidente que resultou em dano real para o paciente ${ }^{2,3}$.

A quimioterapia antineoplásica é uma das terapias mais utilizadas no tratamento do câncer, podendo ser utilizada de forma isolada ou em combinaçáo com outras modalidades de tratamento ${ }^{4}$.

Os quimioterápicos antineoplásicos atuam em nível celular, e interferem no processo de crescimento e divisão celular; logo, para prestar assistência nessa área de atuação, é necessário qualificaçáo da equipe de enfermagem diante das particularidades do mecanismo de ação dos medicamentos, além das possíveis reaçóes decorrentes do tratamento ${ }^{4}$.

A existência ou falhas nos processos que garantem práticas assistenciais seguras propiciam a desconfiança e comprometimento na relação paciente e profissional de saúde; possibilidade de danos psicológicos e sociais; e diminuição da possibilidade de alcançar o desfecho esperado $^{5,6}$.

Os eventos em uma ou mais etapa do processo de administração do medicamento (prescrição, preparação, dispensação e administração) podem ter consequências graves, podendo levar o paciente a óbito ${ }^{7}$.
Diante dessa questáo, o objetivo deste estudo é identificar as estratégias recomendadas na literatura que visam à segurança do paciente na administração de quimioterapia antineoplásica.

\section{MÉTODO}

Trata-se de um estudo de revisão integrativa da literatura que apresenta como objetivo analisar pesquisas relevantes que permitem a síntese de determinado assunto, contribuindo para o aumento da produção do conhecimento e disponibilizando o resultado de várias pesquisas, a partir de um único estudo.

Para elaborar a revisão integrativa, foram percorridas as seguintes etapas: identificação do tema, busca na literatura, categorização dos estudos, avaliação dos estudos incluídos na revisão integrativa, interpretação dos resultados e síntese do conhecimento evidenciado nos artigos analisados.

Assim, diante da importância da adoção de medidas preventivas que possam diminuir os riscos da utilização dos quimioterápicos para os pacientes com câncer, surgiu a seguinte questão norteadora: Quais as estratégias recomendadas nas produçóes científicas para a segurança na administração de quimioterapia antineoplásica?

A busca dos artigos foi realizada de abril a agosto de 2013 mediante consulta às bases de dados, Cumulative Index to Nursing and Allied Health Literature (CINAHL) e US National Library of medicine (PubMed). Utilizou-se como estratégia de busca na PubMed os termos: Antineoplastic Agents"[mh] OR Antineoplas*[tiab] OR chemotherap*[tiab] OR "Medication Therapy Management"[mh] OR "administration and dosage"[sh])) AND ((Medication Errors[mh] OR Medication Errors[tiab]) AND (nursing[sh] OR nursing[tiab] OR oncologic nursing[mh])); na CINAHL: os descritores patient safety and error medication or patient safety and error medication and agents antineoplastic. Os artigos foram selecionados pelos descritores do DeCSdescritor em Ciências da Saúde do Portal BVS, e do MeshMedical Subject Heading do PubMed.

Os critérios de inclusão foram: publicações em português, inglês e espanhol disponíveis, gratuitamente, nas referidas bases de dados, textos completos no período de 2000 a 2013, que abordavam, em seu resumo, as intervençôes/estratégias para administração segura de medicamentos antineoplásicos. Foram excluídos da pesquisa Teses e Dissertaçôes, bem como artigos que não abordavam a temática proposta.

Para extração, apresentação e análise de dados dos artigos, foi utilizado um instrumento contemplando os itens: ano, periódico, título, objetivos, metodologia e nível de evidência.

A determinação do nível de evidência foi baseada no sistema de hierarquia de evidências classificados como 
nível 1: evidências de metanálide ou revisão sistemática de estudos controlados; nível 2: evidências de estudos clínicos randomizados e bem delineados; nível 3: evidências de estudos clínicos não randomizados; nível 4: evidências de estudos de coorte e caso-controle; nível 5: evidências de revisão sistemática de estudos descritivos; nível 6: evidências de um único estudo descritivo ou qualitativo; nível 7: evidências de estudos com parecer ou relatórios de autoridades e/ou especialistas.

Inicialmente, fez-se uma pré-seleçáo dos artigos localizados nas buscas efetuadas por meio da leitura dos títulos e resumo, lidos separadamente, por duas pesquisadoras de forma independente, visando ao refinamento dos resultados. Quando surgiram dúvidas em relação ao conteúdo do trabalho, estes foram préselecionados para posterior análise. Após a seleção dos artigos, foi realizada uma segunda análise pelo par de revisoras com a leitura aprofundada dos artigos préselecionados, obedecendo aos critérios estabelecidos e que atendiam ao objetivo deste estudo.

Os artigos foram organizados por data de publicação do mais recente para o mais antigo e posterior à sua leitura, a amostra foi agrupada em categorias, conforme o enfoque principal identificado.

\section{RESULTADOS E DISCUSSÃO}

De acordo com as estratégias pré-definidas na metodologia utilizada nesta pesquisa, a busca resultou em 1.385 publicaçôes científicas. Foram encontradas 1.204 publicaçóes na CINAHL, 181 na PUBMED. Após a leitura, foram selecionadas 16 publicaçóes de língua inglesa, sendo: 9 na CINAHL, 7 na PUBMED. Entretanto, em virtude da duplicação nas bases de dados, foram excluídas mais três publicaçôes da base Pubmed. Considerando-se a base de dados na primeira pesquisa, manteve-se como referência aquela em que as publicaçóes científicas apareciam pela primeira vez na ordem de busca.
$\mathrm{Na}$ Tabela 1, encontram-se as publicações científicas utilizadas e distribuídas de acordo com: ano, periódico, base de dados, título, objetivos, autores, metodologia, e nível de evidência científica.

Nas publicaçóes analisadas, os autores têm como foco os protocolos, procedimentos e a fase de administração de quimioterápicos, destacando-se a prescrição na qual é necessária a validaçáo tanto pelo enfermeiro como pelo farmacêutico, preparação/diluição, administraçáo, e educação dos pacientes. Estabelecendo condutas únicas, de forma clara, para a manutenção da segurança em todas as etapas no processo de quimioterapia.

Os protocolos de agentes antineoplásicos tornam-se cada vez mais complexos e há possibilidade da ocorrência de erros em cada etapa do processo, com os profissionais envolvidos, assumindo responsabilidades cada vez maiores, para favorecer a manutençáo da segurança do paciente. Os erros podem estar relacionados com práticas profissionais e procedimentos errôneos e os sistemas, incluindo o próprio processo ou todas as suas etapas desde a prescrição até a administração, educação e o monitoramento. Dessa forma, devem ser elaborados e utilizados protocolos, a fim de que haja uniformidade nas condutas e procedimentos, para prestar um cuidado eficaz e seguro ${ }^{7-9}$.

A prescrição de quimioterápicos deve ser realizada de forma eletrônica, por médicos especialistas, contendo nome genérico dos antineoplásicos, identificaçâo do paciente, cálculo das doses, limites de dosagem, via de administração, ordem e velocidade de infusão $0^{6,7,10-13}$. Sáo recomendaçôes simples utilizadas para minimizar a probabilidade de um incidente ocorrer ao paciente e propiciar o aumento na segurança do paciente.

Entre as recomendaçôes, destacam-se: prescriçôes de quimioterápicos realizadas por médicos qualificados; elaboração de protocolos, preferencialmente informatizados, incluindo doses, duração e objetivos da terapia, inserindo também os protocolos de pesquisa clínica; preferência por prescriçóes eletrônicas, contendo

Tabela 1. Artigos selecionados

\begin{tabular}{|c|c|c|c|c|c|c|c|}
\hline Ano & Periódico & Base & Tí́tulo & Objetivo & Autores & Método & $\begin{array}{l}\text { Nível de } \\
\text { evidência }\end{array}$ \\
\hline 2013 & $\begin{array}{l}\text { Journal of } \\
\text { Oncology } \\
\text { Practice }\end{array}$ & CINAHL & $\begin{array}{l}\text { Chemotherapy Administration } \\
\text { Safety Standards Including } \\
\text { Standards for the Safe } \\
\text { Administration and Management of } \\
\text { Oral Chemotherapy }\end{array}$ & $\begin{array}{l}\text { Descrever normas } \\
\text { para a segurança } \\
\text { na administração de } \\
\text { quimioterápicos incluindo } \\
\text { via oral }\end{array}$ & $\begin{array}{l}\text { NEUSS,M.N, } \\
\text { POLOVICH M, } \\
\text { MCNIFF KK, et al. }\end{array}$ & $\begin{array}{l}\text { Revisão de } \\
\text { literatrura }\end{array}$ & 6 \\
\hline 2012 & $\begin{array}{l}\text { Oncology } \\
\text { Nursing Forum }\end{array}$ & CINAHL & $\begin{array}{l}\text { Revisions to the } 2009 \text { American } \\
\text { Society of Clinical Oncology/ } \\
\text { Oncology Nursing Society } \\
\text { Chemotherapy Administration } \\
\text { Safety Standards: Expanding the } \\
\text { Scope to Include Inpatient Settings. } \\
\text { Oncology Nursing Forum }\end{array}$ & $\begin{array}{l}\text { Analisar o processo que } \\
\text { inicialmente desencadeou } \\
\text { as normas de segurança } \\
\text { em quimioterapia e } \\
\text { justificar as mudanças } \\
\text { realizadas }\end{array}$ & $\begin{array}{l}\text { JACOBSON JO, } \\
\text { POLOVICH M, } \\
\text { GILMORE TR, } \\
\text { et al. }\end{array}$ & $\begin{array}{l}\text { Parecer de } \\
\text { especialistas }\end{array}$ & 7 \\
\hline
\end{tabular}


Tabela 1. continuação

\begin{tabular}{|c|c|c|c|c|c|c|c|}
\hline Ano & Periódico & Base & Título & Objetivo & Autores & Método & $\begin{array}{l}\text { Nível de } \\
\text { evidência }\end{array}$ \\
\hline 2011 & $\begin{array}{l}\text { BMJ Quality and } \\
\text { Safety }\end{array}$ & PUBMED & $\begin{array}{l}\text { Safety culture in healthcare: a } \\
\text { review of concepts, dimensions, } \\
\text { measures and progress }\end{array}$ & $\begin{array}{l}\text { Definir a cultura de } \\
\text { segurança, identificar } \\
\text { teorias e rever seu } \\
\text { desenvolvimento }\end{array}$ & $\begin{array}{l}\text { HALLIGAN M, } \\
\text { ZECEVIC A. }\end{array}$ & $\begin{array}{l}\text { Revisão de } \\
\text { literatura }\end{array}$ & 6 \\
\hline 2010 & $\begin{array}{l}\text { European } \\
\text { Journal of } \\
\text { Cancer Care }\end{array}$ & CINAHL & $\begin{array}{l}\text { Medication errors in chemotherapy: } \\
\text { incidence, types and involvement of } \\
\text { patients in prevention: A review of } \\
\text { the literature }\end{array}$ & $\begin{array}{l}\text { Identificar erros na } \\
\text { administração de } \\
\text { quimioterápicos e } \\
\text { informar sobre a } \\
\text { participação dos pacientes } \\
\text { na prevenção de erros }\end{array}$ & $\begin{array}{l}\text { SCHWAPPACH, } \\
\text { DL, WERNLI M. }\end{array}$ & $\begin{array}{l}\text { Revisão de } \\
\text { literatura }\end{array}$ & 6 \\
\hline 2010 & $\begin{array}{l}\text { Oncology } \\
\text { Nursing Forum }\end{array}$ & PUBMED & $\begin{array}{l}\text { Oncology nurses' perceptions about } \\
\text { involving patients in the prevention } \\
\text { of chemotherapy administration } \\
\text { errors }\end{array}$ & $\begin{array}{l}\text { Idenificar a percepção } \\
\text { e a experiência de } \\
\text { enfermeiros oncológicos } \\
\text { sobre } 0 \text { envolvimento } \\
\text { de pacientes na } \\
\text { prevenção de erros em } \\
\text { quimioterapia }\end{array}$ & $\begin{array}{l}\text { SCHWAPPACH DL, } \\
\text { HOCHREUTENER } \\
\text { MA, WERNLI M. }\end{array}$ & $\begin{array}{l}\text { Estudo } \\
\text { descritivo de } \\
\text { abordagem } \\
\text { qualitativa }\end{array}$ & 6 \\
\hline 2009 & ONS Connect & PUBMED & $\begin{array}{l}\text { Prevent inadvertent administration } \\
\text { of intrathecal vincristine }\end{array}$ & $\begin{array}{l}\text { Descrever medidas } \\
\text { para pevenir erros } \\
\text { na administração de } \\
\text { vincristina }\end{array}$ & EISENBERG $S$. & $\begin{array}{l}\text { Revisão de } \\
\text { literatura }\end{array}$ & 6 \\
\hline 2009 & $\begin{array}{l}\text { Journal } \\
\text { of Clinical } \\
\text { Oncology }\end{array}$ & CINAHL & $\begin{array}{l}\text { American Society of Clinical } \\
\text { Oncology/Oncology Nursing Society } \\
\text { Chemotherapy Administration } \\
\text { Safety Standards }\end{array}$ & $\begin{array}{l}\text { Descrever as normas } \\
\text { de segurança na } \\
\text { administraçáa de } \\
\text { quimioterápicos } \\
\text { instituídas pela ASCO }\end{array}$ & $\begin{array}{l}\text { JACOBSON J0., } \\
\text { et al. }\end{array}$ & $\begin{array}{l}\text { Revisão de } \\
\text { literatura }\end{array}$ & 6 \\
\hline 2007 & $\begin{array}{l}\text { Clinical Journal } \\
\text { of Oncology } \\
\text { Nursing }\end{array}$ & CINAHL & $\begin{array}{l}\text { A Model of Chemotherapy } \\
\text { Education for novice oncology } \\
\text { nurses that supports a culture of } \\
\text { safety }\end{array}$ & $\begin{array}{l}\text { Analisar todas as fases do } \\
\text { processo de quimioterapia } \\
\text { e identificar potenciais } \\
\text { erros }\end{array}$ & LEOS NS & $\begin{array}{l}\text { Estudo } \\
\text { descritivo }\end{array}$ & 6 \\
\hline 2007 & ONS Connect & PUBMED & $\begin{array}{l}\text { Chemotherapy education for } \\
\text { novice oncology nurses may create } \\
\text { a culture of safety for nurses and } \\
\text { patients }\end{array}$ & $\begin{array}{l}\text { Descrever o processo } \\
\text { utilizado pelo hospital } \\
\text { para prevenir erros } \\
\text { na administração de } \\
\text { quimioterapia }\end{array}$ & OEITREICHER P. & $\begin{array}{l}\text { Estudo } \\
\text { descritivo }\end{array}$ & 6 \\
\hline 2006 & The Oncologist. & CINAHL & Preventing Chemotherapy Errors & $\begin{array}{l}\text { Revisar os princípios } \\
\text { na administração de } \\
\text { quimioterapia e prevenir } \\
\text { erros }\end{array}$ & SCHULMEISTER, L. & $\begin{array}{l}\text { Revisão de } \\
\text { literatura }\end{array}$ & 6 \\
\hline 2006 & $\begin{array}{l}\text { Clinical Journal } \\
\text { of Oncology } \\
\text { Nursing }\end{array}$ & CINAHL & $\begin{array}{l}\text { Preventing Vincristine } \\
\text { Administration Errors: does } \\
\text { Evidence Support Minibag } \\
\text { Infusions? }\end{array}$ & $\begin{array}{l}\text { Apontar medidas } \\
\text { para prevenir erros } \\
\text { na administração de } \\
\text { vincristina }\end{array}$ & SCHULMEISTER, L. & $\begin{array}{l}\text { Revisão de } \\
\text { literatura }\end{array}$ & 6 \\
\hline 2005 & $\begin{array}{l}\text { Clinical Journal } \\
\text { of Oncology } \\
\text { Nursing }\end{array}$ & CINAHL & $\begin{array}{l}\text { Ten Simple Strategies to Prevent } \\
\text { Chemotherapy Errors }\end{array}$ & $\begin{array}{l}\text { Apontar medidas } \\
\text { para prevenir erros } \\
\text { na administração de } \\
\text { quimioterápicos }\end{array}$ & SCHULMEISTER, L. & $\begin{array}{l}\text { Revisão de } \\
\text { literatura }\end{array}$ & 6 \\
\hline 2002 & $\begin{array}{l}\text { Medication } \\
\text { Misadventures } \\
\text {-Guidelines }\end{array}$ & CINAHL & $\begin{array}{l}\text { Guidelines on Preventing } \\
\text { Medication Errors with } \\
\text { Antineoplastic Agents Medication } \\
\text { Misadventures }\end{array}$ & $\begin{array}{l}\text { Auxiliar pofissionais } \\
\text { de saúde a prevenir } \\
\text { erros durante as } \\
\text { fases do processo de } \\
\text { quimioterapia }\end{array}$ & ASHP & $\begin{array}{l}\text { Parecer de } \\
\text { especialistas }\end{array}$ & 7 \\
\hline
\end{tabular}


as seguintes informaçôes: nome genérico dos agentes antineoplásicos, paciente específico, dados para o cálculo das doses de drogas (altura, peso), resultado de testes laboratoriais, vias de administração, velocidade de infusão e sequência de administração; anamnese incluindo exame físico com peso, altura, avaliação de função específica dos órgãos; avaliação da presença ou ausência de alergias e/ou história de outras hipersensibilidades; limites de dosagem e vias de administraçáo; prescriçóes de quimioterápicos por via intratecal realizadas separadamente das drogas administradas por outras vias; respeito às políticas e normas institucionais; desenvolvimento de um checklist dos itens incluídos nas prescriçōes; prescrições verbais de quimioterápicos apenas para interromper a administração; verificar se as prescriçóes incluem: antieméticos, hidratação, agentes de proteção e fatores de crescimento, quando indicados; eliminação do uso de abreviaturas e inclusão da assinatura do profissional responsável pela prescrição; ajuste das doses somente após comunicaçáo prévia ao paciente.

Tais medidas e recomendaçóes facilitam o trabalho dos profissionais envolvidos, favorecendo que haja a diminuição de falhas entre as etapas integrantes do processo de administração de quimioterápicos, podendo ser consideradas estratégias eficazes para serem utilizadas como forma profilática.

As estratégias específicas para a prescrição de quimioterapia preconizam que devem ser realizadas por médicos qualificados e nunca por residentes ou especializandos, devendo ser confirmada no mínimo por mais um médico especialista, antes de seguir para as demais etapas, observando, principalmente, os protocolos institucionais. Outras propostas são prescriçôes eletrônicas e informatizadas com a utilizaçáo de softwares específicos que somente permitem o término do ato de prescrever, se todos os dados forem preenchidos. Atenta-se para os dados como altura, peso, superfície corporal, dose, data, confirmação de diagnóstico, eliminação do uso de abreviaturas, para que não haja indução ao erro, além da verificação da inclusão de antieméticos, hidratação, agentes de proteção e fatores de crescimento, essenciais em situaçóes de protocolos de alta dose de antineoplásicos que podem acarretar toxicidades severas ao paciente ${ }^{7,12-14}$.

A validaçáo, o preparo e dispensação de quimioterápicos devem ser realizados por profissional capacitado, de acordo com as normas institucionais, farmacêuticos ou enfermeiros oncológicos, seguindo recomendaçóes simples: verificação da prescrição antes da diluição, confirmação de cálculo de dosagens, rotular o quimioterápico após a diluição contendo identificação completa do paciente e antineoplásicos, organizaçáo e boa iluminaçáo do local de trabalho ${ }^{8,9,11,12}$.

Prioriza-se a avaliação da prescrição antes do preparo e administração; checagem do peso e cálculo das dosagens dos quimioterápicos e superfície corporal; rotular imediatamente o quimioterápico após a diluiçáo com nome completo do paciente, número do prontuário e data de nascimento, nome do medicamento genérico, via de administração, volume, data e hora de preparação e expiração da diluição; os quimioterápicos administrados por via intratecal devem ser preparados e armazenados em local diferente dos outros agentes antineoplásicos; rotular com etiquetas de advertência os quimioterápicos que são administrados por via intratecal; preparar vincristina em bolsas de solução salina, mas não utilizar bomba infusora em razáo da maior possibilidade de extravasamento; preparar vincristina em seringas de $10 \mathrm{ml}$; local de trabalho com espaço suficiente e organizado; a dispensação de agentes quimioterápicos por via intratecal deve ser realizada no exato momento que for administrado no paciente; examinar as datas de validade dos frascos, número de registro e o lote das drogas; diluir os medicamentos de acordo com as recomendações dos fabricantes; colocar o quimioterápico diluído em saco plástico para ser transportado ou armazenado de acordo com as recomendaçóes do fabricante ou institucionais ${ }^{8,9,11,13}$.

No Brasil, de acordo com a legislação vigente é privativo ao farmacêutico o preparo dos antineoplásicos e demais medicamentos que possam causar risco ocupacional ao manipulador em estabelecimentos de saúde públicos ou privados ${ }^{15}$.

Sugestôes específicas são utilizadas para a diluição da vincristina como frases de advertência sobre seu uso exclusivo por via intravenosa, visto que sua administração, de forma incorreta, pode levar ao extravasamento ou a óbito. Recomenda-se também a diluição com o uso de seringa de $10 \mathrm{ml}$ ou bolsa de soluçáo salina; entretanto, não há consenso na literatura devido ao risco de aumento de extravasamento. Assim, o profissional deve ficar atento durante a sua administração, por ser uma droga vesicante com alto potencial de dano ao paciente ${ }^{10-12,14}$.

Os profissionais responsáveis pelo preparo de quimioterápicos devem ter atenção especial no quimioterápico vincristina, que, em algumas situaçóes, deve ser preparado em bolsas de solução salina ou seringas de $10 \mathrm{ml}$, apresentando no rótulo advertência de uso exclusivo por via intravenosa. Contudo, não há consenso na literatura quanto ao preparo em bolsa, em razão da possibilidade de aumentar o risco de extravasamento, sendo preferível a utilização em seringas de $10 \mathrm{ml}^{10,16}$.

A administração dos quimioterápicos é a última etapa para evitar que um incidente ocorra ao paciente; entretanto, a segurança do paciente deve ser de responsabilidade de todos os profissionais que estão implicados nesse processo.

Os enfermeiros devem ser qualificados e habilitados para atuar nesta área, tendo conhecimento sobre farmacocinética da droga e protocolos terapêuticos institucionais e de pesquisa, definição de protocolos 
para casos de extravasamento, registro em prontuário de quaisquer intercorrências identificadas pelo enfermeiro ou relatadas pelo paciente, orientaçóes ao paciente pré e pós-quimioterapia e checklist de enfermagem para administraçãó $7,8,11,13,16,17$.

Recomenda-se na etapa de administração: a verificação por dois enfermeiros capacitados e habilitados para identificação do paciente (nome completo, número do prontuário e data de nascimento); checagem se o paciente apresenta pulseira de identificação com pelo menos dois identificadores; revisão de histórico no prontuário de alergias ao quimioterápico; identificação do nome do quimioterápico, dose, volume, via de administração, volume, data de início e término, aparência, integridade física da droga e assinatura de outros profissionais nas etapas anteriores; análise dos registros de tratamento do paciente e se houve intervalo de tempo adequado desde o último tratamento; identificação de toxicidade relacionada ao tratamento; análise das prescriçóes para confirmar se há medicamentos de suporte, antieméticos, hidratação e protetores; recalcular as doses de quimioterapia; comparação dos rótulos dos quimioterápicos que foram diluídos com as prescriçóes e o protocolo proposto para o paciente; verificar se há sequência específica prescrita; ao orientar o paciente fornecer um folder de orientação acerca do tratamento a ser realizado; monitorar o paciente, a fim de evitar incidentes, tais como reação de hipersensibilidade, extravasamento de quimioterapia vesicante e infiltração; fornecer orientação pós-quimioterapia, incluindo autocuidado e monitoramento das reaçôes adversas aos medicamentos; definir condutas para extravasamento de agentes quimioterápicos; o enfermeiro deve ser capacitado e avaliado antes de ser autorizado a administrar quimioterápicos, utilizando conhecimento científico sobre as drogas, intervalos, dosagem adequada, reaçóes adversas a medicamentos, técnicas de administração e manuseio seguro com instrução prática supervisionada; as instituiçôes de saúde devem avaliar os enfermeiros anualmente ou com maior frequência caso ocorra problemas de desempenho; registrar em prontuário os medicamentos antineoplásicos administrados, doses, vias de administração, data e hora de início, a duração ou o tempo que o tratamento foi completado e se foram observados ou relatados pelos pacientes reaçóes adversas aos medicamentos durante ou após administração; políticas e procedimentos para cuidados de suporte a toxicidades e emergências; capacitaçáo de enfermeiros para a administração de quimioterápicos incluindo manuseio, educaçáo do paciente e eventos adversos; avaliar e confirmar a integridade funcional dos dispositivos de acesso vascular, bombas de medicamentos e outros dispositivos; comunicar imediatamente quaisquer erros ao pessoal responsável ou supervisores na utilização de antineoplásicos; cumprir as diretrizes previamente estabelecidas; elaborar checklist de enfermagem para administração de quimioterápicos; vincristina preparada em bolsas de solução salina não deve ser administrada com o auxílio de bomba infusora em razáo da maior possibilidade de extravasamento ${ }^{9,11,15-17}$.

Os profissionais de saúde devem participar da educação dos pacientes, por meio de orientaçôes, contidas em materiais educativos adequados ao seu nível de alfabetização e compreensão, encorajá-los a questionar sobre seu tratamento e padronizar a administração de antineoplásicos, o que permite que o paciente seja capaz de participar ativamente do seu tratamento ${ }^{12,17,18}$.

Entre tais recomendaçóes, apresentam-se: elaboração de materiais educacionais para os pacientes, realizados por profissionais de saúde, disponível nas áreas de aconselhamento e tratamento; padronizaçáo dos procedimentos de administração de quimioterápicos, a fim de que o paciente possa detectar falhas na rotina; encorajar os pacientes a expressar suas preocupaçóes; orientar quanto à administração de quimioterápicos orais: horário, dosagem, descarte, efeitos adversos ${ }^{7,10,11}$.

O envolvimento do paciente em todas as etapas do seu tratamento é importante, uma vez que possibilita a detecção de falhas, tornando-os, corresponsáveis pela sua segurança.

Um novo desafio para os profissionais de saúde é a administração oral de agentes antineoplásicos, em razáo do aumento da participação do paciente nesse processo, dependente do nível de compreensão e assimilação das orientaçôes quanto à autoadministração, bem como a adesáo, monitoramento de eventos adversos e o descarte dos quimioterápicos orais.

A literatura recomenda medidas direcionadas para a educação não só de enfermeiros, mas para todos os profissionais envolvidos por meio da capacitaçáo e avaliaçôes desenvolvidas pelas instituições. Tais profissionais devem demonstrar conhecimento e aptidão em técnicas e procedimentos de segurança na manipulação, administração e descarte, evitando exposição ocupacional ${ }^{7,9,18,19}$.

Entre as estratégias para boas práticas, identificamse: monitoramento de quimioterápicos orais e visitas domiciliares frequentes, definição e utilização de protocolos institucionais, incluindo os de pesquisa clínica, revisão contínua da prescrição em todas as etapas do processo de quimioterapia, comunicação efetiva entre todos os integrantes da equipe de saúde, realização do modelo de falha e análise de efeito (FMEA), prestação de cuidados baseados em evidências, não aceitação de prescriçôes de quimioterápicos por solicitação verbal, análise de protocolos e procedimentos anuais, caso necessário incluindo novas informaçóes e estabelecimento da cultura de segurança baseada na liderança, trabalho em equipe, embasamento em evidências, comunicação, aprendizado, justiça e foco no paciente ${ }^{8,14,19}$. 
As publicaçôes analisadas indicam a necessidade da criação de estratégias para minimizar ou evitar falhas na administração de quimioterápicos a partir de protocolos institucionais para a padronização das condutas e a criação de equipe multidisciplinar para a prevenção e avaliação de erros, além de políticas para monitorar as reaçóes adversas aos medicamentos e a resposta ao tratamento ${ }^{8,9}$.

\section{CONCLUSÃO}

A administração de medicamentos antineoplásicos é uma prática relevante para os enfermeiros que atuam na área da oncologia. Há necessidade do estabelecimento da identificação das atribuiçóes de cada profissional de saúde envolvido no processo de administração de quimioterápicos.

As recomendações nos periódicos internacionais fazem referência à necessidade de diretrizes centradas na responsabilidade do uso de medicação exclusivo para profissionais envolvidos com a fase da prescrição, preparo e administração de quimioterápicos, identificando-se a atuação profissional de médicos, farmacêuticos e enfermeiros.

As publicaçóes científicas analisadas seguem as recomendações da American Society of Health System Pharmacists (ASHP) do ano de 2002, apresentando poucas alteraçóes até o ano de 2013 e somente para quimioterápicos orais e a vincristina.

Somente a utilização de boas práticas não é suficiente para evitar que incidentes na administração de quimioterápicos ocorram. Tornam-se necessárias a criação de uma cultura voltada para o compartilhamento da responsabilidade e a implementação de políticas e normas institucionais, a fim de melhorar a segurança, elaboradas a partir de uma equipe multiprofissional com características interdisciplinares.

\section{CONTRIBUIÇÕES}

Talita dos Santos Ribeiro contribuiu na concepção e planejamento do projeto de pesquisa, na obtenção e análise e interpretação de dados e na redação e revisão crítica. Valdete Oliveira Santos contribuiu na concepção e planejamento do projeto de pesquisa, na obtenção e análise e interpretação de dados e na redação e revisão crítica.

\section{Declaraçáo de Conflito de Interesses: Nada a Declarar. \\ REFERÊNCIAS}

1. The Research Priority Setting Working Group of the World Alliance for Patient Safety. Sumary of the evidence on patient safety: implications for research. Geneva: Word Health Organization; 2008.
2. World Health Organization. Conceptual framework for the international classification for patient safety: version 1.1. Final Technical Report. Geneva: World Health Organization; 2009 Jan.

3. Watcher RM. Compreendendo a segurança do paciente. Porto Alegre: Artmed; 2010.

4. Bonassa EMA, Gato MIR, Mota MLS, Molina P. Conceitos gerais em quimioterapia antineoplásica. In: Bonassa EMA, Gato MIR. Terapêutica oncológica para enfermeiros e farmacêuticos . 4a ed. São Paulo: Atheneu; 2012.

5. Mendes KDS, Silveira RCP, Galvão CM. Revisão Integrativa: método de pesquisa para a incorporação de evidências na saúde e na enfermagem. Texto $\&$ contexto enferm. 2008; 17(4):758-64.

6. American Society of Health System Pharmacists. ASHP Guidelines on Preventing Medication errors with Antineoplastic agents. AM J Health Syst Pharm. 2002;59:164-68.

7. Neuss MN, Polovich M, McNiff K, Esper P, Gilmore TR, LeFebvre KB, et al. Chemotherapy Administration Safety Standards Including Standards for the Safe Administration and Management of Oral Chemotherapy. J Oncol Pract. 2013 Mar;9(2 Suppl):5s-13s.

8. Sheridan-Leos N. A model of chemotherapy education for novice oncology nurses that supports a culture of safety. Clin J Oncol Nurs. 2007 Aug;11(4):545-51.

9. Eisenberg S. Prevent inadvertent administration of intrathecal vincristine. ONS Connect. 2009 Jan;24(1):22.

10. Schwappach DL, Wernli M. Medication errors in chemotherapy: incidence, types and involvement of patients in prevention. A review of the literature. Eur J Cancer Care (Engl). 2010 May;19(3):285-92.

11. Jacobson JO, Polovich M, Gilmore TR, Schulmeister L, Esper P, Lefebvre KB, et al. Revisions to the 2009 american society of clinical oncology/oncology nursing society chemotherapy administration safety standards: expanding the scope to include inpatient settings. J Oncol Pract. 2012 Jan;8(1):2-6.

12. Schulmeister L. Preventing chemotherapy errors. Oncologist. 2006 May;11(5):463-8.

13. Schulmeister L. Preventing vincristine administration errors: Does evidence support minibag infusions? Clin J Oncol Nurs. 2006 Apr;10(2):271-3.

14. Oestreicher P. Chemotherapy education for novice oncology nurses may create a culture of safety for nurses and patients. ONS Connect. 2007 Oct;22(10):26-7.

15. CONSELHO FEDERAL DE FARMÁCIA. Resolução $\mathrm{n}^{\circ} 565$, de 6 de dezembro de 2012.

16. Jacobson JO, Polovich M, McNiff KK, LeFebvre KB, Cummings C, Galioto M, et al; American Society of Clinical Oncology; Oncology Nursing Society. American Society of Clinical Oncology/Oncology Nursing Society 
Chemotherapy Administration Safety Standards. Oncol Nurs Forum. 2009 Nov;36(6):651-8.

17. Schwappach DL, Hochreutener MA, Wernli M. Oncology nurses' perceptions about involving patients in the prevention of chemotherapy administration errors. Oncol Nurs Forum. 2010 Mar;37(2):E84-91.
18. Schulmeister L. Ten simple strategies to prevent chemotherapy errors. Clin J Oncol Nurs. 2005 Apr;9(2):201-5.

19. Halligan M, Zecevic A. Safety culture in healthcare: a review of concepts, dimensions, measures and progress. BMJ Qual Saf. 2011 Apr;20(4):338-43. 


\section{Abstract}

Introduction: Patient's safety can be defined as reducing unnecessary damage, which can occur, associated with health care to a minimum acceptable risk. Errors in one or more steps in the process of chemotherapy can have serious consequences for patients, ranging from unexpected toxicities to death. Objective: Identify in the recommended literature strategies aimed at patient safety in administering antineoplastic chemotherapy. Method: this is an integrative literature review that used the following inclusion criteria: publications in Portuguese, English and Spanish, available for free on the CINAHL and PUBMED databases in full texts in the period of 2000 - 2013. Results: The search resulted in 1385 publications, and selected 13 international scientific publications that met the study objective. Discussion: In the analyzed articles, the authors emphasize the protocols, procedures and chemotherapy administration process, establishing unique behaviors, clearly, to maintain safety at all stages. Conclusion: The use of good practices is not enough to prevent the failures or errors in administering chemotherapeutic drugs. It becomes necessary to create a safety culture focused on sharing responsibility and implementing institutional policies and standards, in order to improve safety, both elaborated from a multidisciplinary team with interdisciplinary features.

Key words: Antineoplastic Agents; Medication Errors; Oncology Nursing; Patient Safety; Review

\section{Resumen}

Introducción: La seguridad del paciente se puede definir como la reducción mínima aceptable del riesgo de daños innecesarios que se producen asociados con el cuidado de la salud. Los errores en uno o más pasos en el proceso de la quimioterapia pueden tener graves consecuencias para los pacientes, desde toxicidades inesperadas hasta la muerte. Objetivo: Identificar las estrategias recomendadas de la literatura encaminadas a la seguridad del paciente en la administración de la quimioterapia antineoplásica. Método: Se trata de una revisión integradora de la literatura que utiliza los siguientes criterios de inclusión: Publicaciones en Portugués, Inglés y Español, disponibles de forma gratuita a partir de la CINAHL y PubMed textos completos en el período 2000-2013. Resultados: La búsqueda resultó en 1385 publicaciones, y seleccionó 13 publicaciones científicas internacionales que cumplieron el objetivo del estudio. Discusión: En los artículos analizados, los autores hacen hincapié en el uso de los protocolos, los procedimientos y los procesos de administración de la quimioterapia, el establecimiento de conductas únicas, de forma clara, para mantener la seguridad en todas las etapas. Conclusión: El uso de las mejores prácticas no es suficiente para evitar fallas o errores se producen en la administración de la quimioterapia. Es necesario crear una cultura de seguridad orientada a la distribución de responsabilidades y la aplicación de las políticas y los acuerdos institucionales con el fin de mejorar la seguridad, desarrollado a partir de un equipo multidisciplinario con características interdisciplinarias. Palabras clave: Antineoplásicos; Errores de Medicación; Enfermería Oncológica; Seguridad del Paciente; Revisión 